\title{
Alcuni teoremi di statica razionale
}

\author{
(del D. ${ }^{r}$ Giuseppe Bardeldi, a Milano.)
}

1. Riferito un sistema di forma invariabile a tre assi ortogonali delle $x, y, z$, siano: $n$ il numero delle forze, $P$ l'intensità di una qualunque tra esse, $x, y, z$ le coordinate del suo punto di applicazione, $\alpha, \beta, \gamma \mathrm{i}$ suoi coseni di direzione. Indichiamo con $X, Y, Z, M_{x}, M_{y}, M_{z}$ le somme delle componenti delle forze secondo gli assi ed i momenti delle coppie componenti poste nei tre piani ortogonali, e si ponga:

$$
\begin{aligned}
& X^{3}+Y^{2}+Z^{2}=R^{2} \\
& X M_{x}+Y M_{y}+Z M_{s}=T \\
& M_{m}=\frac{T}{R} .
\end{aligned}
$$

Il momento risultante $M_{0}$ del sistema corrispondente al punto di coordinate $x_{0}, y_{0}, z_{0}$, ritenute le denominazioni :

$$
\begin{aligned}
& M_{x_{0}}=M_{x}-y_{0} Z+z_{0} Y \\
& M_{y_{0}}=M_{y}-z_{0} X+x_{0} Z \\
& M_{z_{0}}=M_{z}-x_{0} Y+y_{0} X
\end{aligned}
$$

vien dato dall'equazione:

$$
M_{0}^{2}=M_{x_{0}}^{2}+M_{y_{0}}^{2}+M^{2}{ }_{y_{0}} \text {, }
$$

e se chiamiamo $\theta$, l'angolo che l'asse della coppia di momento $M_{0}$ fa con una retta data di cui siano $\lambda, \mu, \nu$ i coseni di direzione, avremo:

$$
M_{0} \cos \theta=\lambda M_{x_{0}}+\mu M_{y_{0}}+\nu M_{z_{0}} .
$$

Se da questa e dalla precedente eliminiamo $M_{0}$, si trova:

$$
\left(M_{x_{0}}^{2}+M_{y_{0}}^{2}+M_{y_{0}}^{2}\right) \cos ^{2} \theta=\left(\lambda M_{x 0}+\mu M_{y_{0}}+\nu M_{x_{0}}\right)^{2} .
$$


Quando si ritengano le $x_{0}, y_{0}, z_{0}$ variabili, questa equazione rappresenta il luogo dei centri di riduzione ai quali corrispondono coppie risultanti i cui assi sono egualmente inclinati alla retta data. Come è facile il provare, il luogo ora determinato è una superficie cilindrica di secondo ordine avente le generatrici parellele alla direzione della forza risultante, e la cui sezione retta è un'ellisse, un'iperbole od una parabola, secondo che l'angolo $\theta$ è minore, maggiore od eguale all' angolo, che il piano perpendicolare alla retta data fa colla direzione della risultante. Quando la retta si prenda parallela alla direzione della risultante, la superficie cilindrica riesce di rotazione, e la sua equazione si deduce dalla precedente ponendo in luogo di $\lambda, \mu, \nu$ rispettivamente $\frac{X}{R}, \frac{Y}{R}, \frac{Z}{R}$, con che si trova

$$
M_{x_{0}}^{2}+M_{y_{0}}^{2}+M_{z_{0}}^{2}=\frac{T^{2}}{R^{2} \cos ^{2} \theta}
$$

il momento risultante:

$$
M_{0}=\frac{T}{R \cos \theta}=\frac{M_{m}}{\cos \theta}
$$

è in questo caso costante per tutti $\mathrm{i}$ punti di una stessa superficie, varia coll'angolo $\theta$ da una superficie all'altra e riceve il valor minimo $M_{m}$ per $\theta=0$. Sottraendo da ambo i membri della (2) la quantità $\frac{T^{2}}{R^{2}}$, e ponendo le denominazioni :

$$
\left.\begin{array}{rl}
R^{2} A & =Y M_{z}-Z M_{y} \\
R^{2} B & =Z M_{x}-X M_{z} \\
R^{2} G & =X M_{y}-Y M_{x}
\end{array}\right\}
$$

si giunge con facili riduzioni alla seguente:

$$
\begin{aligned}
& \left\{\left(y_{0}-B\right) Z-\left(z_{0}-G\right) Y\right\}^{2}+\left\{\left(z_{0}-G\right) X-\left(x_{0}-A\right) Z\right\}^{2} \\
& +\left\{\left(x_{0}-A\right) Y-\left(y_{0}-B\right) Z\right\}^{2}=M^{2}{ }_{m} \operatorname{tang}^{2} \theta .
\end{aligned}
$$

L'asse di questa superficie, o as se centrale del sistema, ha per equazioni:

$$
\frac{x_{0}-A}{X}=\frac{y_{0}-B}{Y}=\frac{z_{0}-C}{Z}
$$

nelle quali si decompone la precedente quando si supponga $\theta=0$. Il raggio $\Delta$ della sezione retta è dato dall' equazione:

$$
R \Delta=M_{m} \operatorname{tg} \theta
$$


e combinando questa colla superiore, che dà il valore di $M_{0}$, si deduce:

$$
M_{0}^{2}=M_{m}^{2}+R^{2} \Delta^{2} \text {. }
$$

Per le cose dette, la proprietà che comunemente viene assunta quale definizione dell'asse centrale di un sistema di forma invariabile, deriva da una proprietà più generale delle coppie risultanti del sistema stesso, giacchè la superficie cilindrica ordinaria di cui il medesimo è asse, e che venne considerata primamente da Pornsot nella sua Statica, è un caso particolare del sistema di superficie cilindriche rappresentate dalla (1).

Per $\theta=\frac{\pi}{2}$ l'equazione (1) si riduce alla seguente:

$$
\lambda M_{x}+\mu M_{y}+\nu M_{x}+x_{0}(\mu Z-\nu Y)+y_{0}(\nu X-\lambda Z)+z_{0}(\lambda Y-\mu X)=0
$$

e quindi il luogo dei centri di riduzione a cui corrispondono coppie risultanti aventi gli assi perpendicolari ad una direzione data è un piano parallelo a questa direzione ed a quella della forza risultante. Se invece supponiamo $\theta=0$, avuto riguardo alla relazione:

$$
\lambda^{2}+\mu^{2}+\nu^{2}=1
$$

la (1) si decompone nelle due:

$$
\frac{M_{x_{0}}}{\lambda}=\frac{M_{y_{0}}}{\mu}=\frac{M_{z_{0}}}{\nu}
$$

ed anche:

$$
\frac{x_{0}-a}{X}=\frac{y_{0}-b}{Y}=\frac{z_{0}-c}{Z}
$$

dove si posto:

$$
\begin{aligned}
& R^{2} a \cos \phi=\mu M_{x}-\nu M_{z} \\
& R^{2} b \cos \phi=\nu M_{y}-\lambda M_{x} \\
& R^{2} c \cos \phi=\lambda M_{x}-\mu M_{z} \\
& \lambda X+\mu Y+\nu Z=R \cos \phi
\end{aligned}
$$

onde il luogo dei centri di riduzione, corrispondentemente ai quali gli assi delle coppie risultanti riescono paralleli ad una retta data, è una retta parallela alla direzione della forza risultante. L'asse centrale puo considerarsi come un caso particolare della retta ora determinata, giacchè dalle equazioni di questa si deducono quelle già trovate, col fare:

$$
\lambda R=X, \quad \mu R=Y, \quad \nu R=Z \text {. }
$$


2. Si conduca pel punto $x_{0}, y_{0}, z_{0}$ la parallela alla retta giả immaginata, si prenda su di essa a partire dal punto in un verso determinato una lunghezza $L$, e si chiami $\Delta$ la minima distanza tra questa retta e l'asse centrale, avremo:

$\Delta R \operatorname{sen} \widehat{R L}=\left(A-x_{0}\right)(Y \nu-Z \mu)+\left(B-y_{0}\right)(Z \lambda-X \nu)+\left(C-z_{0}\right)(X \mu-Y \lambda)$ ossia :

$$
\begin{array}{r}
\Delta R \operatorname{sen} \widehat{R L}=\lambda\left[B Z-G Y-y_{0} Z+z_{0} Y\right]+\mu\left[C X-A Z-z_{0} X+x_{0} Z\right]+ \\
\nu\left[A Y-B X-x_{0} Y+y_{0} X\right] .
\end{array}
$$

Ma dalle equazioni (3) deduconsi facilmente le seguenti:

$$
\left.\begin{array}{l}
B Z-G Y=M_{x}-X \frac{M_{m}}{R} \\
C X-A Z=M_{y}-Y \frac{M_{m}}{R} \\
A Y-B X=M_{z}-Z \frac{M_{m}}{R}
\end{array}\right\}
$$

per le quali la precedente colla sostituzione diviene:

$$
\begin{aligned}
\Delta R \operatorname{sen} \widehat{R L}=\lambda M_{x}+\mu M_{y}+\nu M_{2}+X\left(y_{0} \nu-z_{0} \mu\right)+Y\left(z_{0} \lambda-x_{0} \nu\right)+ \\
\\
Z\left(x_{0} \mu-y_{0} \lambda\right)-M_{m} \cos \widehat{R L},
\end{aligned}
$$

e chiamando $N$ il momento della coppia del sistema, che ha per asse la retta $L$, il cui valore è dato dalla:

$$
N=\lambda M_{x}+\mu M_{y}+\nu M_{x}+X\left(y_{0} \nu-z_{0} \mu\right)+Y\left(z_{0} \lambda-x_{0} \nu\right)+Z\left(x_{0} \mu-y_{0} \lambda\right)
$$

avremo :

$$
N=M_{m} \cos \widehat{R L}+\Delta R \operatorname{sen} \widehat{R L} \text {. }
$$

A questa equazione debbono sempre soddisfare un momento qualunque $N$ del sistema, componente o risultante, e le quantita $\Delta, \widehat{R L}$ che vi si riferiscona, ed essa esprime, che il momento stesso puó sempre considerarsi come risultante dei due momenti posti in piani ortogonali, $M_{m}, R \Delta$. Se perd $N$ è momento risultante, ha luogo la relazione:

$$
N \cos \widehat{L R}=M_{m}
$$


epperò la precedente si riduce a:

$$
\Delta R=N \operatorname{sen} \widehat{R L}
$$

da queste, quadrando e sommando, ottiensi la relazione giả veduta:

$$
N^{2}=M_{m}^{2}+\Delta^{2} R^{2} \text {. }
$$

Dati i momenti di due coppie componenti $N_{1}, N_{2}$, gli angoli $\theta_{1}, \theta_{2}$ che i loro assi fanno coll'asse centrale, e le loro minime distanze $\Delta_{1}, \Delta_{2}$ da questo, purchè $i$ due assi non siano entrambi nè perpendicolari nè paralleli all'asse centrale, si possono sempre assegnare i valori della risultante e del momento minimo. Infatti, per la (6) si hanno le equazioni;

$$
\begin{aligned}
& N_{1}=M_{m} \cos \theta_{1}+\Delta_{1} R \operatorname{sen} \theta_{1} \\
& N_{2}=M_{m} \cos \theta_{2}+\Delta_{2} R \operatorname{sen} \theta_{2}
\end{aligned}
$$

da cui ricavansi $R$ ed $M_{m}$. Se i due momenti dati sono risultanti basta per ciascuno di essi conoscere la minima distanza del suo asse dall'asse centrale, onde avere la forza risultante ed il momento minimo, i quali si dedurranno in tal caso dalle seguenti:

$$
\begin{aligned}
& N_{1}^{2}=M_{m}^{2}+R^{2} \Delta_{1}^{2} \\
& N_{2}^{2}=M_{m}^{2}+R^{2} \Delta_{2}^{2}
\end{aligned}
$$

L'equazione (6) può servire altresì ad assegnare la minima distanza $\Delta$ quando si conoscono le altre quantità che entrano in essa, e quindi anche ad individuare la posizione dell'asse centrale quando per tre coppie distinte si conoscono gli elementi $N, \Delta, \widehat{L R}$ oltre ad $R$ ed $M_{m}$.

3. Date due rette (o forze) $P, Q$ in grandezza, direzione e verso, nonche le posizioni delle rispettive origini, si indichi con:

$$
[P, Q]
$$

il volume del tetraedro costruito sulle medesime, a cui attribuiremo il segno positivo o negativo a seconda che la rotazione segnata da una di essa intorno all'altra, presa come asse della stessa direzione e verso, sia da sinistra a destra o da destra a sinistra, e con $V$ la somma degli $\frac{n(n-1)}{2}$ volumi dei tetraedri, che si ottengono combinando le forze del sistema due a due. Considerando due forze $P_{r}, P_{s}$ qualunque, chiamando $\delta_{r, \&}$ la minima distanza 
tra le loro direzioni, avremo:

$$
\begin{aligned}
& {\left[P_{r}, P_{s}\right]=\frac{1}{6} \cdot P_{r} P_{s} \delta_{r, s} \operatorname{sen} \widehat{P_{r} P_{s}}=\frac{1}{6} P_{r} P_{s}\left\{\left(x_{r}-x_{s}\right)\left(\beta_{r} \gamma_{s}-\beta_{s} \gamma_{r}\right)+\right.} \\
& \left(y_{r}-y_{s}\right)\left(\gamma_{r} \alpha_{s}-\gamma_{s} \alpha_{r}\right)+\left(z_{r}-z_{s}\right)\left(\alpha_{r} \beta_{s}-\alpha_{s} \beta_{r}\right) \xi \text {. }
\end{aligned}
$$

Moltiplicando in ordine le equazioni delle due terne:

$$
\begin{array}{ll}
X=\Sigma P \alpha, & M_{x}=\Sigma P(y \gamma-z \beta) \\
Y=\Sigma P \beta, & M_{y}=\Sigma P(z \alpha-x \gamma) \\
Z=\Sigma P \gamma, & M_{x}=\Sigma P(x \beta-y \alpha)
\end{array}
$$

e sommando i prodotti, si ottiene facilmente:

$$
\begin{aligned}
X M_{x}+Y M_{y}+Z M_{z}=\Sigma P_{r} P_{s}\left\{\left(x_{r}-x_{s}\right)\left(\beta_{r} \gamma_{s}-\beta_{s} \gamma_{r}\right)+\right. & \\
& \left.\left(y_{r}-y_{s}\right)\left(\gamma_{r} \alpha_{s}-\gamma_{s} \alpha_{r}\right)+\left(z_{r}-z_{s}\right)\left(\alpha_{r} \beta_{s}-\alpha_{s} \beta_{r}\right)\right\}
\end{aligned}
$$

dove gli indici $r, s$, diversi tra loro, debbono ricevere tutti i valori $1,2, \ldots$ n. Per la precedente e pel valore di $T$ avremo:

$$
T=6 \Sigma\left[P_{r}, P_{s}\right]=6 V,
$$

relazione la quale dimostra la notissima proprietà del trinomio $T$ di essere indipendente dalla scelta degli assi coordinati. Per la relazione fondamentale, che lega la teoria delle coppie a quella dei momenti, è possibile esprimere il momento $N$ di una coppia qualunque del sistema di forma invariabile mediante le grandezze delle componenti, gli angoli che le loro direzioni comprendono coll' asse della coppia, e le minime distanze $\delta$ tra queste e le direzioni stesse (*); si ha pertanto:

$$
N=\Sigma P \delta \operatorname{sen} \widehat{P L}
$$

cio posto, la equazione trovata nel numero precedente:

$$
N=M_{m} \cos \widehat{L R}+\Delta R \operatorname{sen} \widehat{R L}
$$

si può facilmente tradurre in una relazione tra volumi: moltiplicando per $L$ e richiamando il valore di $M_{m}$, si trova:

$$
L N=\Sigma L P \delta \operatorname{sen} \widehat{L P}=L R \Delta \operatorname{sen} \widehat{L R}+\frac{L T}{R} \cos \widehat{L} \widehat{R}
$$

(*) Brioschi: La Statica dei sistemi di forma invariabile, Cap. III. 
ossia :

$$
6 \Sigma[L, P]=L R \Delta \operatorname{sen} \widehat{L R}+\frac{L T}{R} \cos \widehat{L R} .
$$

Si supponga la risultante diretta secondo l'asse centrale, allora:

e per la (7) risulterà :

$$
L R \Delta \operatorname{sen} \widehat{L R}=6[L, R]
$$

$$
\Sigma[L, P]=[L, R]+\frac{L V}{R} \cos \widehat{L R}
$$

quest'equazione dà il mezzo di calcolare la somma dei volumi dei tetraedri aventi per singoli opposti una retta qualunque e ciascuna forza del sistema, quando si conoscano: il volume del tetraedro costruito sulla retta e sulla risultante, supposta diretta secondo l'asse centrale, e la somma dei volumi dei tetraedri i cui spigoli opposti sono le forze date prese due a due. Accenneremo ad alcune conseguenze a cui conduce la medesima.

Il secondo membro della (8) riceve un valore costante, quando, considerando sempre lo stesso sistema di forze, siano costanti $L, \Delta, \widehat{L R}$, cioè quando $L$ sia presa su una generatrice rettilinea qualunque dell'iperboloide ad una falda di rotazione, che ha per asse l'asse centrale e $\Delta$ per raggio del cerchio di gola; e siccome le generatrici stesse sono assi di coppie risultanti aventi l'egual momento, così possiamo stabilire il seguente teorema: Se in uno qualunque degli assi delle coppie risultanti di egual momento, supposti i medesimi condotti pei rispettivi centri di riduzione, si prende una costante lunghezza (che potrebbe rappresentare il momento risultante), e si costruiscono su di essa e su ciascuna delle date forze come spigoli opposti, i tetraedri, la somma dei volumi di questi sará costante.

Quando la retta $L$ si fa coincidere colla risultante applicata a qualsivoglia centro di riduzione, si ha:

$$
\widehat{L R}=0, \quad[L, R]=0
$$

e quindi in qualunque sistema di forze:

$$
\Sigma[R, P]=V \text {. }
$$

Il secondo membro della equazione trovata, per gli stessi valori di $L, \Delta$, $\widehat{R L}$ mantiene lo stesso valore, se essa si fa sussistere per un altro sistema 
di forze equivalente al primo, giacchè i due sistemi dovranno ammettere la stessa risultante, lo stesso momento minimo e l'identico asse centrale. Sia $Q$ una qualunque delle forze del sistema equivalente, risultera:

$$
\Sigma[L, P]=\Sigma[L, Q]
$$

se le forze $Q$ sono due sole $Q_{1}, Q_{2}$ sarà:

$$
\Sigma[L, P]=\left[Q_{1}, L\right]+\left[Q_{2}, L\right],
$$

e se supponiamo $L$ coincidere con $R$, si ha:

$$
\Sigma[R, P]=\left[Q_{1}, R\right]+\left[Q_{2}, R\right]
$$

e per la $(9)$ :

$$
V=\left[Q_{1}, R\right]+\left[Q_{2}, R\right]=\left[Q_{1}, Q_{2}\right]
$$

nella quale è contenuta la dimostrazione di un notissimo teorema di Chasıres.

Se la retta $L$ e presa in un piano perpendicolare alla risultante, si ha:

$$
\Sigma[P, L]=[R, L]
$$

e quando invece è parallela alla risultante:

$$
\Sigma[L, P]=\frac{L}{R} \cdot V .
$$

La prima di queste relazioni si verifica anche nel caso che il sistema sia riducibile ad una risultante unica e qualunque sia la retta $L$, perchè sappiamo dover allora essere $T=0$ e quindi $V=0$. Allorquando si consideri un sistema in equilibrio, dovendo essere nulli la forza risultante ed il momento minimo, se ne conclude:

$$
[R, L]=0, \quad V=0, \quad \Sigma[L, P]=0 ;
$$

la seconda di queste contiene un conosciuto teorema di Mobrus, e la terza, surrogando ad $L$ successivamente le forze del sistema, fornisce $\boldsymbol{n}$ equazioni, che si verificano nell'equilibrio di qualunque sistema, da me date in altra occasione, ma seguendo un metodo diverso $\left({ }^{*}\right)$, e che vennero in seguito trovate anche dal signor Spotriswoode (**).

3. Immaginiamo un secondo sistema di forma invariabile affatto indipendente dal primo. Siano $Q$ la grandezza di una qualunque delle sue forze,

(*) Rendiconti dell' Istituto Lombardo, 1866, vol. III.

(**) Comptes rendus, 1868, N..$^{\circ} 2$. 
$d$ la minima distanza che la sua direzione ha dalla direzione della forza $P$ qualsivoglia del primo sistema; e per indicare tutti gli altri elementi che si riferiscono al nuovo sistema, ci serviremo delle lettere già usate con analogo significato pel primo sistema, ma accentate. Posto:

$$
W=X M_{x_{1}}+Y M_{y_{1}}+Z M_{z_{1}}+X_{1} M_{x}+Y_{1} M_{y}+Z_{1} M_{z}\left(^{*}\right)
$$

e seguendo lo stesso metodo che ci ha servito al calcolo di $T$, troveremo:

$$
W=\Sigma P Q d \cos P Q=6 \Sigma[P, Q] \text {. }
$$

Il valore di $W$ può essere calcolato altrimenti come segue. Si determini la minima distanza $D$ tra gli assi centrali dei due sistemi: percio comincieremo a porre nella (8) $L=R_{1}, \Delta=D$ ed avremo:

$$
N=D R \operatorname{sen} \widehat{R R_{1}}+\frac{T}{R} \cos \widehat{R R_{1}}
$$

poi per avere $N$, momento della coppia del primo sistema, che ha per asse l'asse centrale del secondo, faremo nella (5) le seguenti sostituzioni

$$
\lambda=\frac{X^{\prime}}{R_{1}}, \mu=\frac{Y_{1}}{R_{1}}, \nu=\frac{Z_{1}}{R_{1}}, x_{0}=A_{1}, y_{0}=B_{1}, z_{0}=C_{1}
$$

e richiamando anche le (4), troveremo:

$$
\begin{array}{r}
N=\frac{1}{R_{1}}\left(X_{1} M_{x}+Y_{1} M_{y}+Z_{1} M_{z}\right)+\frac{X}{R_{1}}\left(M_{x_{1}}-\frac{X_{1} T_{1}}{R_{i}^{i}}\right)+\frac{Y}{R_{1}}\left(M_{x_{1}}-\frac{Y_{1} T_{1}}{R_{i}^{2}}\right)+ \\
\frac{Z}{R_{1}}\left(M_{z_{1}}-\frac{Z_{1}}{R_{1}}\right)=\frac{T_{1}}{R_{1}}-\frac{T_{1}}{R_{1}^{3}}\left(X X_{1}+Y Y_{1}+Z Z_{1}\right)=\frac{W}{R_{1}}-\frac{T_{1}}{R_{1}^{3}} \cos \widehat{R R_{1}}
\end{array}
$$

(*) Se con $M$ ed $M_{1}$ indichiamo $i$ momenti risultanti dei due sistemi corrispondenti all' origine degli assi coordinati, e con $\widehat{M_{1} R}, \widehat{M R_{1}}$ gli angoli che l'asse risultante di un sistema comprende coll'asse centrale dell' altro, al valore di $W$ possiamo dare la seguente forma:

$$
W=M R_{1} \cos \widehat{M R_{1}}+M_{1} R \cos \widehat{M_{1} R}
$$

Questa relazione insieme alla (12)

$$
W=6 \geq[P, Q]
$$

renne data anche dal ch. ${ }^{\circ}$ signor Prof. Chelin nella sua recente memoria: Sulla composizione geometrica dei sistemi di rette, di aree e di punti, pubblicata dall'Accademia di Bologna, e della quale io ebbi comunicazione soltanto quando il mio breve lavoro era gia compilato. La seconda poi di quelle relazioni trovasi altresi dimostrata, come vennemi avvertito dal Prof.: Cremona, in una anteriore memoria dello stesso signor Prof. Cheniñ : Sugli assi centrali delle forze e delle rotazioni nell'equilibrio e nel moto dei corpi (Bologna, 1866). 
e finalmente, eliminando $N$ colla superiore:

$$
W=D R R_{1} \operatorname{sen} \widehat{R R_{1}}+\cos \widehat{R R_{1}}\left(\frac{R T_{1}}{R_{1}}+\frac{R_{1} T}{R}\right) .
$$

Eguagliando i due valori trovati di $W$ ed introducendo la notazione dei volumi, abbiamo:

$$
\Sigma[P, Q]=\left[R, R_{1}\right]+\cos \widehat{R R_{1}}\left(\frac{R V_{1}}{R_{1}}+\frac{R_{1} V}{R}\right)
$$

Quest'equazione dimostra il seguente teorema: Dati due sistemi qualunque di forma invariabile, per calcolare la somma dei volumi dei tetraedri che si possono costruire combinando ciascuna forza dell'uno con ciascuna forza dell'altro, basta conoscere il vo. lume del tetraedro, che ha per spigoli opposti le due risultanti, supposte dirette secondo i rispettivi assi centrali, e le somme dei volumi dei tetraedri che si ottengono combinando in ciascun sistema le forze due a due.

Se si applica l'equazione (9) a ciascuno dei due sistemi prendendo per retta $L$ successivamente le risultanti $R_{1} R$, si hanno le seguenti equazioni:

$$
\begin{aligned}
& \Sigma[R, P]=\left[R, R_{1}\right]+\frac{R_{1}}{R} V \cos \widehat{R R_{1}} \\
& \Sigma\left[R_{1}, Q\right]=\left[R, R_{1}\right]+\frac{R}{R_{1}} V_{1} \cos \widehat{R R_{1}}
\end{aligned}
$$

sommando membro a membro ed avendo riguardo alla (12), si trova:

$$
\Sigma[P, Q]+\left[R, R_{1}\right]=\Sigma\left[R_{1}, P\right]+\Sigma[R, Q] \text {. }
$$

$\grave{E}$ facile enunciare il teorema contenuto in questa equazione: Dati due sistemi affatto indipendenti di forze, la somma dei volumi dei tetraedri, che nascono dal combinare ciascuna forza dell'una con ciascuna dell'altro, più il volume del tetraedro costruito sulle due risultanti drette secondo i rispettivi assi centrali, eguaglia la somma dei tetraedri che si ottengono combinando ciascuna forza di un sistema colla risultante dell'altro, e viceversa. Deriviamo alcuni corollari.

Quando l'uno dei due sistemi, per es. il secondo, è in equilibrio si ha:

$$
R_{1}=0, \quad\left[R, R_{1}\right]=0, \quad \Sigma\left[R_{1} P\right]=0, \quad \Sigma\left[R_{1}, Q\right]=0
$$


e quindi anche:

$$
\Sigma[P, Q]=0
$$

teorema di Chasies (*).

Se i due sistemi sono riducibili ad un'unica risultante:

$$
\Sigma[P, Q]=\left[R, R_{1}\right],
$$

e se inoltre le direzioni delle risultanti si incontrano, sarà :

$$
\left[R, R_{1}\right]=0, \quad \Sigma\left[R_{1}, P\right]=0, \quad \Sigma[R, Q]=0
$$

e quindi ancora:

$$
\Sigma[P, Q]=0
$$

e per conseguenza:

$$
\left.W=0 \quad{ }^{* *}\right) \text {. }
$$

Supponiamo il secondo sistema equivalente al primo, dovranno verificarsi le condizioni :

$$
R=R_{1}, \widehat{R R_{1}}=0, \cdot V=V_{1}, \quad\left[R, R_{1}\right]=0,
$$

ed allora dalla (13) deduciamo:

$$
\Sigma[P, Q]=2 V \text {. }
$$

Consideriamo in particolare il sistema di due forze dirette secondo due rette reciproche ed equivalente al dato; chiamando $Q_{1}, Q_{2}$ le intensità delle due risultanti parziali, avremo:

$$
\Sigma\left[P, Q_{1}\right]+\Sigma\left[P, Q_{2}\right]=2 V
$$

e per la relazione (11) già trovata al numero precedente:

$$
\Sigma\left[P, Q_{1}\right]+\Sigma\left[P, Q_{2}\right]=2\left\{\left[Q_{1}, R\right]+\left[Q_{2}, R\right]\right\}=2\left[Q_{1}, Q_{2}\right] ;
$$

possiamo pertanto concludere col seguente teorema: Ridotto un sistema di forma invariabile a due sole forze agenti secondo due rette reciproche, la somma dei $2 n$ volumi dei tetraedri che si ottengono combinando ciascuna delle componenti con ciascuna delle risultanti parziali, è uguale al doppio del volume del tetraedro avente per spigoli opposti queste ultime.»

(*) Journal de Liouville, tome XII, pag. 213.

(**) BrioscuI: La Statica dei sistemi di forma invariabile, Cap. VII. 
Notiamo infine, che la relazione (13) stabilita indipendentemente dalla (9) comprende questa stessa come caso particolare, alla quale si riduce quando si consideri il secondo sistema costituito di una sola forza $Q$, e si ponga:

$$
Q=L, \quad R_{1}=L, \quad V_{1}=0
$$

cosichè l'equazione:

$$
\Sigma[P, Q]=\left[R_{1}, R\right]+\cos \widehat{R R_{1}}\left\{\frac{R V_{1}}{R_{1}}+\frac{R_{i} V}{R}\right\}
$$

è la più generale fra le analoghe che possono dare relazioni di volumi tetraedri che risultano dalla considerazione simultanea di due sistemi di forze: da essa infatti si possono derivare tutti $i$ teoremi dimostrati negli ultimi due numeri precedenti.

Milano, gennajo 1871. 